\title{
Author Correction: Reconstructing quantum states with generative models
}

Juan Carrasquilla (D, Giacomo Torlai, Roger G. Melko and Leandro Aolita

Correction to: Nature Machine Intelligence https://doi.org/10.1038/s42256-019-0028-1, published online 11 March 2019.

In the version of this Article originally published, the following sentence in the Acknowledgements was incorrect: 'Research at Perimeter Institute is supported through Industry Canada and by the Province of Ontario through the Ministry of Research \& Innovation. It should have read: 'Research at the Perimeter Institute is supported by the Government of Canada through Innovation, Science and Economic Development Canada and by the Province of Ontario through the Ministry of Economic Development, Job Creation and Trade.' This has now been corrected.

Published online: 22 March 2019

https://doi.org/10.1038/s42256-019-0045-0 